\title{
La educación física en educación primaria: espacio de construcción de las masculinidades y feminidades Physical Education in primary education: space for the construction of masculinity and femininity Iriana Sánchez-Alvarez, Carmen Rodríguez-Menéndez, Omar García-Pérez Universidad de Oviedo (España)
}

Resumen: El artículo muestra los resultados de una investigación que ha estudiado la construcción de las identidades de género en la escuela primaria. Más específicamente, nuestro objetivo ha sido comprender e interpretar el papel de las clases de Educación Física como contextos de interacción que permiten resistirse/acomodarse a las personalidades de género hegemónicas. Durante una estancia de seis meses, para realizar una investigación etnográfica, se realizó una observación participante en un aula de sexto curso compuesto por 13 niñas y 13 niños. El trabajo de campo se completó con seis grupos de discusión y doce entrevistas individuales. Los resultados permiten concluir que la actividad física en general, y el futbol en particular, son elementos articuladores de la jerarquía de género en la escuela determinando el estatus que exhiben los niños con una masculinidad hegemónica. También se concluye que las clases de educación física son espacios privilegiados de interacción de género, en las que la actitud del profesorado favorece el mantenimiento de las categorías normativas de género.

Palabras clave: Educación Física, menores, escuela, etnografía, género.

Abstract: This article shows the results of a research that has studied the construction of gender identities in primary schools. More specifically, our objective was to understand and to interpret the role of Physical Education classes as an interaction context that allows resisting/accommodating hegemonic gender personalities. During a six-month stay, in order to perform ethnographic research, we observed 13 girls and 13 boys from a sixth-grade group. The fieldwork was completed with six focus groups and twelve individual interviews. The results allow us to conclude that physical activity in general, and football in particular, are articulating elements of gender hierarchy in schools by determining the status shown by children with a hegemonic masculinity. As a conclusion, physical education classes are privileged spaces for gender interaction, in which the attitude of teachers stimulate the maintenance of gender normative categories.

Key Words: Physical Education, child, school, ethnography, gender.

\section{Introducción}

En el ámbito anglosajón diversas investigaciones han analizado la construcción de las identidades de género en la escuela desde el feminismo postestructuralista. Desde este paradigma la identidad de género no es fija, sino que es precaria, contradictoria y se negocia y renegocia constantemente a través de la interacción social. No obstante, también se reconoce que no todas las masculinidades y feminidades tienen el mismo valor social. De forma que los modelos de deseabilidad social coinciden con el arquetipo de la masculinidad y la feminidad hegemónica. En este marco, la escuela puede ayudar o entorpecer en el desarrollo de las categorías normativas de género al crear un espacio en el que se ponen en juego diversos recursos que permiten crear una jerarquía de género.

En este contexto, investigaciones realizadas en España y en países anglosajones han concedido una especial relevancia al deporte en general, y al fútbol en particular, como un recurso de gran valor en la formación de la masculinidad hegemónica y en la configuración de la jerarquía de género en las escuelas (Hernández, Vidiella, Herráiz \& Sancho, 2007; Vidiella, Herráiz, Hernández \& Sancho, 2010). Los varones deben mostrar un cuerpo musculado y tonificado que demuestre que se es bueno ejercitando los deportes percibidos como masculinizantes, pues niños y niñas también afirman que el ballet, la danza, el vóleibol o la gimnasia rítmica son deportes femeninos, mientras que el levantamiento de pesas, el fútbol, el boxeo o el kárate son masculinos (Blández, Fernández \& Sierra, 2007; Schmalz \& Kerstetter, 2006).

Quienes muestran mayor velocidad, fuerza, violencia o

Fecha recepción: 23-09-19. Fecha de aceptación: 11-12-19

Carmen Rodríguez Menéndez habilidad, especialmente en el fútbol, se encuentran en la cúspide de la jerarquía de género (Atencio \& Koca, 2011; Campbell, Gray, Kelly \& Maclsaac, 2016; Jeanes, 2011; Swain, 2003, 2006). Además, también suelen ser los más populares del aula quienes lideran las clases de educación física y quienes controlan los juegos y deportes (Parker \& Curtner-Smith, 2012; Soler, 2009). Ese ejercicio de dominación también se exhibe espacialmente porque la zona lúdica está copada por el deporte, más concretamente por el fútbol (Clark \& Paechter, 2007; Epstein, Kehily, Mac an Ghaill \& Redman, 2001; Garay, Vizcarra \& Ugalde, 2017).

En este sentido, diversas investigaciones también indican que se estigmatiza y humilla a los varones que no son buenos deportistas, a quienes no les gustan los deportes típicamente masculinos, a quienes participan en deportes femeninos o a aquellos que juegan al fútbol con las niñas (Atencio \& Koca, 2011; Fagrell, Larsson \& Redelius, 2012; Martino \& Pallotta-Chiarolli, 2006; Mayeza, 2017; Parker, 1996; Parker \& Curtner-Smith, 2012; Schmalz \& Kerstetter, 2006; Swain, 2000, 2002). De forma que la subordinación se realiza en términos de diferencia y cualquier aspecto contrario a la pauta dominante es utilizado en contra de estos niños.

Así, los varones que carecen de habilidades para determinados deportes son acosados verbalmente por el grupo, llamándoles gays, maricas o afeminados (Atencio \& Koca, 2011; Parker, 1996; Swain, 2003, 2006; Mayeza, 2017). La homofobia se usa para controlar y regular la conducta de los niños situándolos en una jerarquía que subordina a quienes manifiestan comportamientos «no masculinos» o «femeninos» (Vidiella, et al., 2010, p.105). Así, Silva, Botelho-Gomes y Vilodre (2012) concluyeron que las actitudes homofóbicas subyacían en la resistencia verbal de los chicos a realizar ejercicios de gimnasia rítmica en las clases de educación física puesto que asumían que un cuerpo masculino era in- 
capaz de realizar los movimientos flexibles y elegantes asociados a este deporte (véase también Blández, et al., 2007). En el estudio de Mayeza (2017) también se constató que los niños no querían participar en deportes «femeninos» por miedo a ser adjetivados como «maricas».

En lo relativo a la construcción de las feminidades, el deporte escolar también determina la posición subordinada de las chicas en la jerarquía de género. Las actividades deportivas, especialmente el fútbol, son consideradas terreno masculino que está vetado para las chicas (Jeanes, 2011; Renold, 1997). Los estudios confirman que las niñas son excluidas de los deportes en general, y del fútbol en particular, porque los niños opinan que no tienen las destrezas necesarias para jugar bien (Atencio \& Koca, 2011; Fagrell, et al., 2012; Swain, 2006), proclamando que es un terreno que les compete exclusivamente a ellos y que no desean compartir con las chicas (Epstein, et al., 2001), hasta el punto que aquellos niños que no ostentan una masculinidad hegemónica critican esta «intromisión» para alinearse con sus compañeros hegemónicos (Clark \& Paechter, 2007). Por todo ello los estudios indican que es habitual que las chicas tengan que pedir autorización a los chicos para poder jugar con ellos (Clark \& Paechter, 2007; Renold, 1997).

Asimismo los estudios sobre el fútbol escolar indican que cuando unos y otras juegan juntos es habitual que ellos se muevan libremente por el terreno de juego, cuestionando las habilidades de las chicas cuando tocan el balón o realizan algún pase. Por otra parte, ellas tienden a agruparse en una posición defensiva, quejándose de que los niños las ignoran y no les pasan el balón (Atencio \& Koca, 2011; Clark \& Paechter, 2007; Fagrell, et al., 2012; Soler, 2009). Como argumenta Soler (2009) esta situación provoca que las niñas concluyan que no saben jugar, adoptando distintos roles que las excluyen del juego. Así, se dedican a prestar atención a cuestiones que no son del juego, a charlar con las compañeras, a inventarse nuevos ejercicios o a modificar la tarea para que les resulte más interesante. Este distanciamiento de la práctica deportiva también se observó en el estudio de Clark y Paechter (2007) pues las niñas se desvinculaban de la práctica deportiva bromeando sobre la seriedad con la que los niños se tomaban el fútbol y otros deportes.

No obstante, no todas las niñas se acomodan a los patrones de feminidad hegemónica, pues hay quienes participan activamente en las clases de educación física y no tienen miedo de ostentar una posición de dominio en los deportes. Ahora bien, la feminidad de estas niñas es cuestionada, constatándose que quienes juegan al fútbol, con intensidad y dedicación, son consideradas «marimachos»o lesbianas (Clark \& Paechter, 2007; Paechter \& Clark, 2007; Parker \& Curtner-Smith 2012; Mayeza, 2017). Por esta razón, Jeanes (2001) concluyó que algunas niñas de 10 y 11 años de su estudio mantenían un comportamiento de género transgresor al jugar al fútbol pero también estaban constreñidas por discursos más tradicionales, cultivando una apariencia física muy femenina. Al mismo tiempo, las niñas eran reacias a «jugar como niños», evitando la agresividad y el contacto físico, y criticando a las que manifestaban tales comportamientos.

Finalmente, debemos mencionar que los estudios indican que el profesorado de educación física juega un papel primordial en el mantenimiento del orden social de género. Su implicación puede tomar la forma de comentarios irónicos, miradas o reproches hacia conductas no adecuadas al estereotipo (Clark \& Paechter, 2007; Parker \& Curtner-Smith, 2012; Rodríguez \& García, 2009; Silva, et al., 2012). En otros casos, mantienen una actitud pasiva durante el desarrollo de las prácticas deportivas sin promover, de forma activa, la interacción entre géneros (Fagrell, et al., 2012). En ocasiones, se culpabiliza, de forma indirecta, a las niñas al manifestar que «no quieren jugar» o que deben ser más asertivas y dinámicas en el espacio de juego (Clark \& Paechter, 2007; Soler, 2009), mientras se sigue manteniendo el prejuicio de la inevitabilidad biológica de las conductas que manifiestan unos y otras (Fagrell, et al., 2012; Garay, et al., 2017; Parker \& Curtner-Smith, 2012).

\section{Material y método}

\section{Objetivos, método y participantes}

Los resultados de este artículo forman parte de una investigación etnográfica que ha tenido por objetivo comprender e interpretar las características que tienen los contextos de interacción de género en la escuela primaria. Más específicamente, mostramos aquellos resultados que tienen que ver con el uso del deporte escolar como estrategia clave en la configuración de las masculinidades y las feminidades, así como el papel de las clases de educación física en el desarrollo de las dinámicas de poder entre géneros.

El material de análisis proviene de una etnografía realizada durante un curso académico, desde mediados del mes de noviembre hasta mediados del mes de mayo, en un centro público y urbano de educación primaria del Principado de Asturias localizado en un contexto socioeconómico y cultural medio-bajo. Por la situación del barrio donde se sitúa el centro escolar se entrelazan familias inmigrantes, especialmente de países latinos y eslavos, así como familias españolas de clase media y trabajadora. Asimismo, algunos niños y niñas procedían de casas de acogida.

Todas las semanas se acudía al centro durante tres días, previamente pactados con la dirección, y después de haber obtenido el consentimiento informado de las familias. Tras los primeros días de estancia en el campo se decidió realizar la observación participante en un grupo de sexto de primaria. Las disciplinas académicas a las que se tuvo acceso eran aquellas impartidas por la tutora y por el docente de Educación Física. Esta decisión se tomó puesto que algunos miembros del profesorado no estaban cómodos con la presencia en sus clases de una observadora durante tanto tiempo. Por todo ello, las asignaturas en las que se realizó la observación participante fueron matemáticas, lengua castellana, educación física, así como en los recreos. La clase estaba compuesta por 26 estudiantes, 13 niñas y 13 niños. Asimismo, también pueden ser considerados participantes del estudio la tutora del grupo que impartía las clases de matemáticas y lengua castellana, así como el docente de educación física, al estar ambos físicamente presentes en el momento de las observaciones.

Instrumentos de recogida de datos

Los datos fueron recogidos usando tres instrumentos. 
En primer lugar, se realizó una observación participante en la que la investigadora explicó a la comunidad educativa su papel como observadora que, de vez en cuando, participaría en ciertas actividades, aunque de forma moderada. Esta situación ayudó a la investigadora a mantenerse entre la «familiaridad» y el «extrañamiento» (Velasco \& Díaz de Rada, 2004). De este modo, estaba sentada cerca de los niños y las niñas para poder escuchar y observar de forma directa, pero sin estar dentro de sus grupos de trabajo para no afectar a sus comentarios, opiniones o comportamientos. La información observada era recogida, in situ, en las notas de campo para, posteriormente, ser detallada y ampliada en el diario de campo.

La información obtenida a través del diario de campo se completó con la recogida a través de grupos de discusión y entrevistas individuales. El grupo de discusión es una técnica de conversación y narración, lo que resulta muy interesante para una investigación escolar cuyos protagonistas principales son los niños y las niñas. Se organizaron cuatro grupos de discusión con seis personas cada uno. Fue necesario el uso de una grabadora para registrar los datos y transcribirlos llegado el momento del análisis. Para evitar que los niños y las niñas preparasen sus respuestas, no se les mostraron las preguntas ni el tema de discusión, simplemente se les explicó qué era una discusión, las normas, el respeto por las opiniones ajenas y la solicitud del turno de palabra. De igual forma se afirma que quienes integran un grupo de discusión deben tener algo en común para hablar, con comodidad, de ciertos temas controvertidos (Dorio, Sabariego \& Massot, 2004). Intentamos respetar al máximo esta indicación pero con ciertos límites. Si bien, en algunos casos, nuestros grupos estaban formados por personas afines, no siempre fue posible porque hubieran quedado excluidos algunos niños y niñas, normalmente quienes ya lo estaban en el día a día dentro del aula.

De forma complementaria, se realizaron doce entrevistas semiestructuradas flexibles y dinámicas (Angrosino, 2012). Los niños y las niñas entrevistadas habían oído, sentido y vivido situaciones que nos interesaba conocer y de las que no habíamos podido participar. Ellos y ellas nos comunicaron, con su propia experiencia, aquellos hechos sociales en los que participaban y que eran de nuestro interés para la temática objeto de estudio. Durante las entrevistas, por la edad y características del alumnado, fue necesario clarificar algunas de las cuestiones. En ocasiones, cuando la conversación se separaba del camino deseado, se les señalaba o llamaba la atención sobre algunos aspectos importantes para que opinasen sobre ellos. En estas entrevistas fue muy importante realizar una escucha activa, esforzándonos por comprender lo que los niños y las niñas estaban expresando.

\section{Procedimiento de análisis de datos}

El material recabado es discursivo y narrativo, por lo que se decidió emplear un proceso de análisis de datos que se ajustase a estas características. El análisis de contenido cualitativo se inició con el análisis del texto recogido a través del diario de campo, los grupos de discusión y las entrevistas individuales, manteniendo su carácter textual y categorizando el contenido de todos los documentos. Para realizar esta categorización se estableció un código para las unidades de contenido, dando una denominación común o código a un conjunto de fragmentos de texto que compartían una misma idea.

Una vez que tuvimos el sistema de categorías con toda la información clasificada en ellas (véase figura 1), pasamos a realizar el análisis descriptivo/interpretativo. Para ello, partimos de los textos ya categorizados y procesados y de ellos se infirieron enunciados descriptivos. Finalmente, estos enunciados descriptivos fueron interpretados y explicados a partir del contraste con la literatura existente.

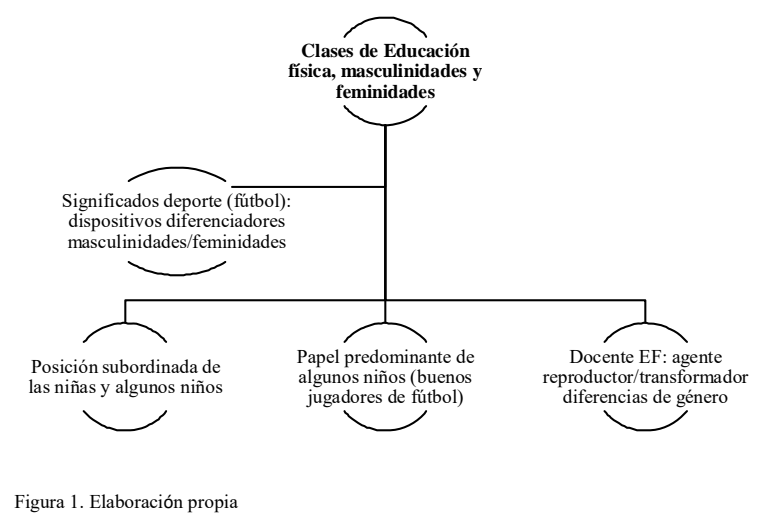

Resultados

Los significados sociales otorgados al deporte, especialmente al fútbol

En las conversaciones mantenidas, las palabras feminidad y masculinidad fueron asociadas, por la mayoría de niños y niñas, al aspecto físico, las aptitudes y las aficiones, incluidas las deportivas. En relación a éstas, muchos niños y niñas asumieron, con naturalidad y desde el desconocimiento, que los deportes considerados femeninos, tales como el ballet, eran inferiores porque opinaban que no se necesitaban especiales habilidades para su aprendizaje y ejecución, razón por la cual no había que entrenar ni esforzarse. Además, con frecuencia, recurrieron a diferencias biológicas para justificar que niños y niñas prefiriesen distintos deportes:

n1-(niño) «mira las guajas son tranquilas y les gustan las cosas para presumir [risas] ... pintarse y la ropa esa brillante, casi no tienen que entrenar porque eso de bailar, saltar o volteretas se les da bien, para el fútbol o entrenas y vas a gimnasio o nada, no puedes jugar...»

Entrevistadora «¿es más fácil el ballet que el fútbol?»

n2-(niño) «somos más fuertes desde el nacimiento [risas] y bueno, hay que correr y llevar golpes, el fútbol no les gusta a las guajas, empezarían a llorar y gritar...[niñas protestan] no protestéis anda que es verdad...»

n 4-(niño) «tú sabes lo que tienen que entrenar los de fútbol y esas cosas, vale sí en ballet también pero no es igual, yo creo que no es tan duro, por eso hay que estar fuerte para fútbol...»

n 5-(niña) «yo creo que los nuestros son más fáciles, bueno es más bien de cada uno, vale, igual los deportes de chico son más físicos» (2GD).

Como podemos observar, tanto niños como niñas manifiestan claros prejuicios sexistas respecto a las posibilidades y potencialidades de unos y otras para distintos deportes, a 
la par de un profundo desconocimiento de las habilidades necesarias para ejecutar, con dominio y pericia, cualquier práctica deportiva. Unos y otras asumen estos prejuicios con naturalidad porque están expuestos, constantemente en su medio social, a mensajes sexistas respecto a la menor capacidad física de las niñas para los deportes y al escaso prestigio social que tienen las actividades deportivas realizadas por mujeres.

En este contexto, los discursos respecto al fútbol, como deporte de gran relevancia en la sociedad española, jugaron un papel muy relevante en la configuración del dualismo de género. Así, este deporte fue usado discursivamente como dispositivo para diferenciar la masculinidad hegemónica de otras masculinidades y de las feminidades. Es más, en deportes como el fútbol se hace gala de necesitar actitudes específicas de los hombres, por ser un deporte impulsivo y duro. En estos fragmentos de una conversación sobre fútbol con niños y niñas, explican sus razones para demostrar por qué es masculino el fútbol:

Entrevistadora-») ¿por qué dices que el fútbol es cosa de tíos? Si hay equipos femeninos y chicas que juegan al fútbol aquí en el colegio...»

n1(niño)-»porque es así de siempre, ahora juegan guajas pero ahora, y en equipos de ellas...el fútbol que se sigue por todos es de tíos ...[sonriendo mira a su grupo] mira, tienes que dar patadas, te dan alguna, te empujan, te tiran, te insultan, tienes que correr mogollón y en sitios que flipas, barro y de todo, te lo digo que yo juego, no es para guajas, seguro que lloran o algo»

n2(niño)-»>creo que es demasiado de tíos....iimaginas a Yaiza jugando, llevando patadas o quitándole un balón a uno? ... [risitas] yo no, tienes que correr y no tener miedo a llevarlas, y al darlas que tienes que empujar y dar alguna patada pa quitar el balón. Mejor jugar cada uno con cada uno [por sexos,] sino problemas fijo...»

Entrevistadora- Que digan algo las niñas ¿es el fútbol un deporte masculino?

n 4(niña)-»bueno mira pues sí, yo paso de que me anden pegando golpes, pero la verdad es que hay niñas que sí juegan eh Jaime, y juegan bien, igual te daban una paliza y todo listo...[Jaime protesta] ya bueno pero eso, creo que sí es más de tíos porque necesitas estar fuerte...» (3GD).

Podemos percibir que el futbol es una excusa para oponer la masculinidad hegemónica a la feminidad. Algunos niños, más que las niñas, asumen como propios los estereotipos sexistas que impregnan la sociedad patriarcal en la que vivimos y no conciben que ellas puedan participar en esta actividad deportiva y, además, hacerlo con habilidad y destreza. Las razones que aportan son de carácter físico-»ellas no son fuertes»-, emocional- «seguro que lloran si reciben un golpe»-, o aludiendo a la normalidad o naturalidad- «siempre ha sido así, es lo normal o lo natural»-. Razones todas ellas que asumen la biologización estereotipada de las diferencias entre géneros $\mathrm{y}$, por tanto, su inevitabilidad. Además, cuando se comentaba el caso de alguna niña que jugaba bien al fútbol, se trató como una excepción y la consideraron «poco femenina»- marimacho-; mostrándose, nuevamente, los prejuicios sexistas de algunos alumnos y alumnas:

n3(niño)-»hay una niña en $4^{\circ}$ que juega bien al fútbol, es como un chico (entre risas) un poco $<$ marimacho $>$, no hace lo de las chicas, bueno es una chica como un chico...»

n4 (niña)-»del fútbol pasamos, menos algunas niñas que son buenas en fútbol pero no me llevo con ellas, son $<$ raras $>$, bueno son amigas de los chicos también» (3GD).

En este contexto, para la mayoría de los niños de nuestra aula el fútbol era un asunto muy serio que ocupaba gran parte de su tiempo libre. Es más, varios de ellos llegaban cada lunes hablando de sus éxitos futbolísticos o de los partidos que habían jugado el fin de semana; además, en los recreos todos los chicos de la clase, sin excepción, jugaban al fútbol. En más de una ocasión, la tutora nos comentó que cuando se dialogaba en el aula sobre el tema del fútbol era necesario cortar la conversación porque o se hacía interminable o se terminaba gritando y con insultos. Muchos chicos de la clase desarrollaban su masculinidad a través del sentido y significado que el fútbol aportaba a sus vidas. Así, los alumnos que se encontraban en la cúspide de la jerarquía de género- Miguel, Jaime o Sebas- eran muy buenos futbolistas. Sus compañeros y compañeras dicen de ellos:

De Sebas: «es buen tío y además es un crack en el fútbol», «sí Sebas mola, no veas cómo juega, es una pasada, quiere ser profesional y todo y puede eh...», «no se mete con nadie, es buen tío, buenísimo en el deporte, me cae bien».

De Jaime y Miguel: «pues de mis compañeros es Miguel y es también Jaime que son amigos. Bueno creo que son los más conocidos de la clase, juegan fútbol muy bien», «Miguel y Jaime, son lo más...y juegan genial a fútbol y son como unos capitanes del grupo» (Extractos de distintas entrevistas individuales).

Como podemos apreciar, estos chicos eran muy populares en la clase y su masculinidad hegemónica era visible, se hacía notar. Mediante la actividad futbolística, estos niños exhibían actitudes de la masculinidad más arquetípica como la competitividad, la agresividad o la fuerza física.

\section{La clase de educación física como contexto de interacción reproductor de la jerarquía de género}

En el contexto de nuestro trabajo de campo, los procesos de interacción en la clase de educación física eran desiguales, pues la mayoría de las niñas no participaban, de forma plena, en las actividades. Además, eran el blanco de las burlas o riñas de muchos de sus compañeros varones, especialmente de quienes adoptaban un papel protagonista. El sentimiento de no pertenencia de las niñas era tan significativo que la mayoría ni siquiera protestaba ante situaciones injustas ni reivindicaba su lugar en las actividades. Sin lugar a dudas, y como se puede ver en los siguientes fragmentos del diario de campo, la violencia verbal, a través de insultos y vejaciones, que exhibían muchos estudiantes varones era frecuente en estas clases y actuaba como un elemento disuasorio que impedía una participación más activa de muchas de las niñas. Y es que a lo largo de todo el trabajo de campo no dejó de sorprendernos la violencia verbal a la que eran sometidas las niñas, y algunos niños no hegemónicos, por parte de un colectivo importante de niños varones. Niños que asumían, sin problemas, los prejuicios sexistas del contexto social en el que vivían y que materializaban en las clases de Educación Física, quizás por ser un ambiente menos estructurado y porque la práctica deportiva es un elemento diferenciador muy relevante: 
«En una de las actividades de hoy, se establecen grupos, siempre desiguales, dado que es el alumnado quien los decide, llevaban jugando unos minutos y empezaron los conflictos, algunos niños insultaban a una niña porque pasaba los aros muy despacio, ella les gritó y en un momento parecía una batalla. El profesor toca el silbato y les manda parar, pero cada vez que pueden se insultan o empujan. $<$ Eres una inútil tía, que es solo saltar aros joer, vamos a perder...joer $>$, $<$ me dejas en paz, voy como quiero guaje $>,<$ anda vete a la mierda $>,<$ vete tú subnormal $>$... Las niñas protestan en voz alta y sus compañeros, entre risas y burlas, les dicen cosas como $<$ anda calla pesada que estás siempre igual $>$. Nunca parece terminar la clase de educación física sin serios conflictos entre niños y niñas».

«En la clase de Educación Física los chicos tratan a sus compañeras de tontas o inútiles y parece que a ellas les da igual, parece que no están allí o que les da igual la clase.... Olaya termina llorando ante los insultos constantes de Jaime que considera que no está jugando bien. Jaime, un vez terminado el juego, le comenta a otros niños, buscando su apoyo, que hay niñas que juegan bien pero la mayoría escapan de la pelota y juegan fatal, además $<$ siempre están llorando $>$. En esta clase ni una sola de las niñas, ni siquiera las más deportistas, mostraron interés por el juego y lo único que hicieron fue formar grupos y hablar, como si no estuviesen allí» (Fragmentos del diario de campo).

En resumen, en el escenario de nuestro trabajo etnográfico la disciplina de educación física fue la materia donde más claramente se solidificaron los roles de género y donde se manifestó, con rotundidad, el ejercicio de poder de un grupo de niños varones. Algunos chicos se sentían poderosos, fuertes y competitivos porque podían demostrar sus cualidades masculinas ante otros niños no hegemónicos, sus compañeras y el profesor. Por otra parte, la mayoría de las niñas asumían un rol de sumisión e incompetencia en las clases, dedicándose a hablar, pasear o manifestar una actitud de desenganche respecto de la actividad que se implementaba.

Asimismo, estas dinámicas de poder claramente desiguales y discriminatorias, eran reforzadas por la actitud del profesor que actuaba como elemento perpetuador de las identidades de género hegemónicas. Así, de su discurso se infería que esperaba de su alumnado unas conductas específicas acomodadas al patrón de género. Era frecuente que les recriminase con frases sexistas: «te parece normal que una niña haga eso», «eso no son cosas de paisano», «no ves que ellos son nenos, pa qué te metes», «es una cría ¿qué quieres, hacerla llorar?, no seas burro hombre» (extractos del diario de campo). También eran habituales sus afirmaciones respecto a la falta de capacidad deportiva de las niñas: «los guajes, lo normal, el fútbol, que es lo que les gusta a ellos, las crías son distintas, les gusta estar tranquilas, bailar sí y esas cosas, alguna es más activa, pero en general pasan bastante del deporte» (Extracto del diario de campo). El docente concluye que hay una distinta preferencia lúdica, que esto ha pasado siempre y que es imposible de modificar.

En suma, manifestaba un comportamiento sexista y estereotipado que determinaba, con claridad, su comportamiento en clase. Así, adoptaba una estrategia de no intervención, limitándose a recriminar determinadas conductas. Como po- demos observar en el siguiente fragmento del diario de campo, y que refleja una situación típica que se observó repetidamente, el profesor anima a que se formen grupos mixtos pero su intervención casi finaliza con esta recomendación. De este modo, la clase acaba convertida en una confusa amalgama de recriminaciones y reproches, interviniendo finalmente para dejar claro que las niñas son menos habilidosas y que los niños tienen que mostrar una actitud «caballerosa» para con ellas:

«En clase de educación física el maestro les propone un juego por equipos en el que deben encestar la pelota en unas cajas. Antes de empezar comenzaron las protestas por parte de algunos niños como Samuel: <jolines menudo rollo, podíamos jugar al fútbol o algo ¿no? $>$, pero el profesor le ignora. El profesor les dice $<$ venga poneros por equipos...tenéis que elegir a alguna de las niñas jeh $i$ que nos conocemos y luego las dejáis solas y pierden siempre, venga o sino os coloco yo $j \mathrm{eh}_{i}>$. Entonces algunos niños, $<$ escogieron $>$ a las niñas que ellos consideran son mejores deportistas y se $<$ deshicieron $>$ de algunos de sus amigos, los que ellos llaman $<$ mantas $>$, para que fuesen al equipo de las niñas. Durante todo el juego, como es habitual, los niños criticaban a las niñas $<$ muévete anda $>$, <joer tía no vales para nada $>$. También el maestro les decía que se moviesen o que tenían que intentarlo, etc., los comentarios del profesor a los niños eran < oye a las niñas no se les dicen esas cosas, que son niñas, piensas que estás en el campo de fútbol $>$, <ayuda a tu compañera hombre, no ves que no sabe $>$, con las niñas muestra siempre una actitud condescendiente como si no fuesen capaces de hacer deporte» (Extracto del diario de campo).

Al mismo tiempo, las alumnas se quejaban constantemente de la pasividad de su maestro ante la actitud agresiva y disruptiva de algunos de sus compañeros varones, revelando su malestar ante esta situación: «Entrevistadora: < ¿por qué estáis tan enfadadas? >-Olaya: <pues es que encima Rafa no hace nada, pa qué se lo vamos a decir, si ya lo ve él y pasa...>» (Extracto del diario de campo). Era frecuente que las niñas declarasen su incomodidad ante esta situación de forma que, a muchas de ellas, esta asignatura les disgustaba por la actitud de su docente y de sus compañeros varones:

n1-(niña)-»la que menos, la educación física, fijo que no me gusta, me gusta bailar y patinar y eso, pero en esa clase no hacemos nada que me guste, son solo deportes de niños y ellos pasan de nosotras, dicen que no servimos para nada y esas historias, buff un asco...»

n2- (niña)- «La que menos me gusta es educación física, no soy buena en el deporte y se monta mucho lío siempre, Rafa no riñe, deja que hagan lo que quieren» (1GD).

En oposición, la mayoría de niños varones disfrutaban de estas clases porque estaban cómodos con la permisividad del docente hacia las conductas irrespetuosas que dirigían a sus compañeras o a otros niños no hegemónicos:

n1- (niño)-»a mí me gusta educación física porque me gusta hacer deporte y Rafa nos deja jugar con el balón y aprendemos a jugar al vóleibol y cosas, además es bueno, castiga poco...»

n2-(niño)-»la que me gusta es educación física porque Rafa mola, nos deja tranquilos y no nos aburrimos, está muy guay...»(2GD).

Las niñas y los niños tenían la sensación de que las 
reprimendas del profesorado nunca tenían consecuencias por lo que muchos niños percibían que tenían carta blanca para actuar como querían. Esta actitud de pasividad mostraba su connivencia y complicidad con las actitudes machistas de ciertos alumnos varones, lo que reforzaba el comportamiento de éstos, al tiempo que demostraba sus propios prejuicios sexistas. Como es evidente por lo desarrollado hasta este momento, ni adoptaba medidas para trabajar la igualdad de género ni desarrollaba una estrategia inclusiva en su programación didáctica.

Por otro lado, hemos de señalar que durante un breve periodo de tiempo hubo un profesor sustituto y la dinámica de interacción fue completamente diferente. El profesor paraba la clase cada vez que aparecían conductas agresivas o faltas de respeto entre unos y otras, parecía sentirse incómodo ante las relaciones conflictivas que definían el día a día de las relaciones entre niños y niñas. Los días que el profesor sustituto se encargó de las clases se hicieron juegos de equipo y configuraba personalmente los grupos $\mathrm{y}$, pese a que parte del alumnado protestó continuamente, al final las clases fueron menos violentas y muchas niñas estuvieron más contentas, al menos no protestaron por tener que ir a la clase de educación física. Para la mayoría de los niños la situación fue diferente, muchos estaban enfadados porque no les gustaba el profesor sustituto, dado que no podían jugar al fútbol durante las clases. Tanto las niñas como los niños sabían que su profesor habitual volvería, así que muchas de ellas comentaban: «las clases así molan, jugar a otras cosas y eso, además al profe no le mola que los guajes se pasen...pero Rafa vuelve y ya sabes...» (Extracto del diario de campo). En el siguiente extracto del diario de campo puede entenderse esta afirmación:

«En este grupo los niños y las niñas suelen estar separados. En el caso de educación física el maestro (sustituto) les pide que formen dos grupos para realizar una actividad y la clase, de forma inmediata y sin ninguna duda, se divide por sexos. El maestro se da cuenta y pregunta: $<$ ¿por qué os colocáis así?>. Entonces decide hacer algo al respecto, lo que desconcierta a niños y niñas acostumbrados a que nadie lo tenga en cuenta» (Extracto del diario de campo).

De este fragmento textual podemos concluir que la actitud del profesorado en las clases de Educación Física es de vital importancia porque o puede ayudar en el mantenimiento y reproducción de los estereotipos de género o puede favorecer el cambio hacia el desarrollo de roles más equitativos.

\section{Conclusiones}

En nuestro estudio etnográfico la actividad física en general, y el futbol en particular, actúan como elementos articuladores de la jerarquía de género en la escuela. De forma similar a otras investigaciones (Blández, et al., 2007; Schmalz \& Kerstetter, 2006), de los discursos de niños y niñas se concluye que tienen ideas muy claras y sexistas respecto a las actividades deportivas, indicando que hay deportes «masculinos»y «femeninos». Deportes que no gozan del mismo prestigio social porque opinan que solamente aquellos que son masculinizantes requieren de habilidades y destrezas específicas, que se logran con un entrena- miento duro y constante (Atencio \& Koca, 2011; Fagrell, et al., 2012; Swain, 2006). Asimismo, el fútbol es el deporte masculino por excelencia, hasta el punto que aquellas niñas que frecuentan su práctica son calificadas de «raras» o «marimachos» (véase también Clark \& Paechter, 2007; Mayeza, 2017); mientras que los niños más hábiles en su práctica ostentan una clara posición de poder en el aula, tal y como también se ha demostrado en otros estudios (Atencio \& Koca, 2011; Campbell, et al., 2016; Jeanes, 2011; Swain, 2003, 2006). En suma, de nuestro trabajo de campo se puede concluir que niños y niñas manifiestan actitudes sexistas respecto a la práctica deportiva, siendo un claro reflejo de aquellas que se muestran constantemente en la sociedad patriarcal en la que viven y conviven.

De forma complementaria, la clase de educación física es el espacio privilegiado donde se ponen en juego las dinámicas de relación entre ambos géneros. Dinámicas muy conflictivas caracterizadas porque los niños, especialmente aquellos que ostentan una masculinidad hegemónica, son protagonistas absolutos, marcando con su comportamiento los espacios y tiempos de lo que ocurre en el gimnasio y otros espacios escolares (véase también Clark \& Paechter, 2007; Epstein, et al., 2001; Garay, et al., 2017; Parker \& CurtnerSmith, 2012; Soler, 2009). Así, a través de los insultos a sus compañeras, por considerar que no tienen habilidades ni destrezas físicas, se configura una dinámica de poder que las relega a un rol alternativo, pasivo y secundario, tal y como diversos autores y autoras ya han concluido para el caso de la posición ostentada por las niñas en la práctica del fútbol (Atencio \& Koca, 2011; Clark \& Paechter, 2007; Fagrell, et al., 2012; Soler, 2009). El ejercicio de esta violencia verbal, que se muestra a través de insultos y vejaciones continuas, demuestra cómo las actitudes machistas se incardinan en los comportamientos de un colectivo de varones de la clase que reproducen el discurso patriarcal.

En ocasiones, ellas se rebelan ante el ejercicio de este poder pero con pocas garantías de éxito porque no cuentan con el apoyo del docente, quien, con su actitud sexista, no entiende, o no quiere entender, los procesos discriminatorios que se desarrollan en sus clases. Así, sus ideas preconcebidas acerca de las distintas conductas y actitudes que tienen niños y niñas se revelan en comentarios muchas veces irónicos (véase también Clark \& Paechter, 2007; Parker \& CurtnerSmith, 2012; Rodríguez \& García, 2009; Silva, et al., 2012) y en otros marcadamente deterministas (Fagrell, et al., 2012; Garay, et al., 2017; Parker \& Curtner-Smith, 2012). A estos comentarios, y de forma similar a otros estudios (Fagrell, et al., 2012), se debe señalar su clara pasividad ante lo que ocurre en sus clases que no hace sino alimentar unas dinámicas de poder altamente conflictivas entre unos y otras, reforzando la jerarquía de género. Si bien es cierto que regaña con frecuencia a los niños por su comportamiento, tampoco hace nada más, por lo que los niños han aprendido que si bien se enfada mucho nunca hace nada y las niñas han asimilado que no pueden contar con su ayuda para acabar con una situación claramente perjudicial para ellas.

Sin lugar a dudas, la construcción de unas dinámicas de relación entre géneros que sean más respetuosas pasa necesariamente por una intervención positiva del profesorado, tal y como se demuestra con los pequeños cambios acaeci- 
dos en el grupo con la llegada de un profesor sustituto visiblemente contrario a mantener el statu quo. En línea similar, Clark y Paechter (2007) relatan la intervención positiva que tuvo, en la escuela donde realizaron su etnografía, un profesor que facilitó una participación activa y en pie de igualdad de las niñas en equipos mixtos de fútbol. Igualmente, Chen y Cutner-Smith (2013) describen la experiencia coeducativa implementada por dos docentes de educación física quienes realizaron un esfuerzo consciente por modificar las dinámicas de poder entre los géneros. Para ello organizaron una propuesta pedagógica destinada a empoderar a los niños y niñas con menos destrezas físicas, modificando las reglas del juego para lograr una mayor equidad y proveyendo a ambos géneros de un acceso igualitario a los mejores tiempos y espacios para la práctica deportiva. Asimismo, distribuyeron equitativamente las responsabilidades en el terreno de juego, reforzando la idea de que todas las posiciones de juego eran importantes. Finalmente, mantuvieron conversaciones con su alumnado para analizar el sexismo imperante en el deporte, reflexionando sobre su propia práctica.

En línea similar, señalamos la propuesta de Rodríguez y Miraflores (2018) respecto a la modificación de las normas del fútbol para hacer más inclusivas las clases de educación física. Esta innovación se acompañó de una serie de recomendaciones para el profesorado, tales como el uso de un lenguaje no sexista o la mención constante a la igualdad de género para acabar con actitudes machistas y estereotipadas en el alumnado. Sobre este último punto, resaltamos, a modo de ejemplo, la experiencia pedagógica desarrollada por Monforte y Úbeda-Colomer (2019) con alumnado de bachillerato en la que se usó un vídeo sobre estereotipos de género en el deporte para provocar una dinámica de debate y diálogo sobre igualdad de género en la práctica deportiva.

De forma complementaria, Paechter (2013) indica la necesidad de introducir en los currícula de la asignatura de educación física la práctica de deportes feminizantes, tal y como ella expone con el ejemplo del ballet y la danza, y en línea similar a la propuesta de Beltrán, Sierra, Jiménez, GonzálezCutre, Martínez y Cervelló (2017) de promocionar en los recreos actividades físico-deportivas que impliquen una mayor participación de las chicas tales como saltar o bailar. Del mismo modo, y ya en el año 2000 Skelton hacía una recomendación respecto a las prácticas deportivas y los juegos discriminatorios, impulsando la idea de que era preciso potenciar la participación de las niñas en todos los deportes y evitar el fomento de una imagen masculina de la actividad física. Creemos que es absolutamente necesario impulsar y mejorar la imagen de las niñas como deportistas capaces tanto desde la visión de los niños como desde la de las propias niñas y del profesorado.

Siguiendo con las recomendaciones es aconsejable que el profesorado garantice la posibilidad de liderazgo tanto de los niños como de las niñas, proponiendo actividades de juego diferentes que no promuevan la competición sino la cooperación, siendo ésta una estrategia que favorece el desarrollo de la inclusividad en el aula, tal y como han señalado diversas investigaciones (Beltrán et al., 2017; Canales, Aravena, Carcamo-Oyarzun, Lorca \& Martínez-Salazar, 2018; Rodríguez \& Miraflores, 2018). De modo que se hace preciso articular también otra forma de evaluar los procesos de ense- ñanza-aprendizaje en las clases de Educación Física que vaya más allá del «deporte de competición» (Monforte \& ÚbedaColomer, 2019, p.78).

Evidentemente para lograr que los maestros y maestras modifiquen su comportamiento y actitud en el gimnasio es necesario que comprendan los procesos de construcción social de las identidades femeninas y masculinas en la escuela y su papel en estos procesos. Solamente a partir de esta sensibilización se podrán llevar a cabo intervenciones satisfactorias que ayuden a modificar las dinámicas de poder y la jerarquía de género en las aulas (Silva, et al., 2012). Para ello un instrumento necesario es la presencia, en el Proyecto Educativo de Centro, de planes o proyectos coeducativos que ayuden a sensibilizar a toda la comunidad educativa respecto a la igualdad de género.

Finalmente, debemos indicar algunas limitaciones del estudio. La información ha sido obtenida a partir de la observación participante realizada por la investigadora y de las entrevistas y grupos de discusión con el alumnado. De modo que hubiese sido útil contrastar y triangular estos datos con la información recogida, por ejemplo, sobre el programa de educación física que planifica y lleva a la práctica el docente. Asimismo, también hubiera sido deseable entrevistar al docente de la asignatura Educación Física para determinar su posición respecto a la influencia de la escuela en los procesos de construcción de las feminidades y masculinidades.

\section{Referencias}

Angrosino, M. (2012). Etnografía y observación participante en la investigación cualitativa. Madrid: Morata.

Atencio, M., \& Koca, C. (2011). Gendered communities of practice and the construction of masculinities in Turkish physical education. Gender and Education, 23, 59-72.

Beltrán, V. J., Sierra, A. C., Jiménez, A., González-Cutre, D., Martínez, C. \& Cervelló, E. (2017). Diferencias según género en el tiempo empleado por adolescentes en actividad sedentaria y actividad física en diferentes segmentos horarios del día. Retos, 31, 3-7.

Blández, J., Fernández, E., \& Sierra, M.A. (2007). Estereotipos de género, actividad física y escuela. La perspectiva del alumnado. Profesorado. Revista de Curriculum y Formación del Profesorado, 11, 1-21.

Campbell, D., Gray, S., Kelly, J., \& Maclsaac, S. (2016). Inclusive and exclusive masculinities in physical education: a Scottish case of study. Sport, Education and Society, 23, 216-228.

Canales, P., Aravena, O., Carcamo-Oyarzun, J., Lorca, J., \& Martínez-Salazar, C. (2018). Prácticas pedagógicas que favorecen u obstaculizan la inclusión educativa en el aula de educación física desde la perspectiva del alumnado y profesorado. Retos, 34, 212-217.

Chen, Y., \& Curtner-Smith, M. (2013). Hegemonic masculinity in sport education: case studies of experienced in-service teachers with teaching orientation. European Physical Education Review, 19, 360-380.

Clark, S., \& Paechter, C. (2007). Why can't girls play football?: gender dynamics and the playground. Sport, Education and Society, 12, 261-276.

Dorio, I., Sabariego, M., \& Massot, I. (2004). Características 
generales de la metodologiìa cualitativa. En R. Bisquerra (Coord.), Metodologiìa de la investigacioìn educativa (pp. 275-292).Madrid: Muralla.

Epstein, D., Kehily, M., Mac an Ghaill, M., \& Redman, P. (2001). Boys and girls come out to play: Making masculinities and femininities in school playgrounds. Men and Masculinities, 4, 158 - 172.

Fagrell, B., Larsson, H., \& Redelius, K. (2012). The game within the game: girl's underperforming position in Physical Education. Gender and Education, 24, 101-118.

Garay, B., Vizcarra, M.T., \& Ugalde, A.I. (2017). Los recreos, laboratorios para la construcción social de la masculinidad hegemónica. Teoría de la Educación. Revista Interuniversitaria, 29, 185-209.

Hernández, F., Vidiella, J., Herráiz, F., \& Sancho, J.M. (2007). El papel de la violencia en el aprendizaje de las masculinidades. Revista de Educación, 342, 103-125.

Jeanes, R. (2011). I'm into high heels and make up but I still love football: exploring gender identity and football participation with preadolescent girls. Soccer \& Society, 12, 402-420.

Martino, W., \& Pallota-Chiarolli, M. (2006). Pero, ¿queì es un chico? Aproximacioìn a la masculinidad en contextos escolares. Barcelona: Octaedro.

Mayeza, E. (2017). Girls don’t play soccer: children policing gender on the playground in a township primary school in South Africa. Gender and Education, 29, 476-494.

Monforte, J. \& Úbeda-Colomer, J. (2019). 'Como una chica': un estudio provocativo sobre estereotipos de género en educación física. Retos, 36, 74-79.

Paechter, C. (2013). Girls and their bodies: approaching a more emancipatory physical education. Pedagogy, Culture \& Society, 21, 261-277.

Paechter, C., \& Clark, S. (2007) Learning gender in primary school playgrounds: findings from the Tomboy Identities Study. Pedagogy, Culture \& Society, 15, 317-331.

Parker,A. (1996). The construction of masculinity within boys' physical education. Gender and Education, 8, 141-157.

Parker, M., \& Curtner-Smith, D. (2012). Sport education: a panacea for hegemonic masculinity in physical education or more of the same? Sport, Education and Society, 17, 479-496.

Renold, E. (1997). 'All they've got on their brains is football. ì Sport, masculinity and the gendered practices of playground relations. Sport, Education and Society, 2,
$5-23$.

Rodríguez, H., \& García,A. (2009). Asimilación de códigos de género en las actividades del recreo escolar. Revista Interuniversitaria de Formación del Profesorado, 64, 59-72.

Rodríguez, L. \& Miraflores, E. (2018). Propuesta de igualdad de género en Educación Física: adaptaciones de las normas en fútbol. Retos, 33, 293-297.

Schmalz, D., \& Kerstetter, D. (2006). Girlie girls and manly men: children's stigma consciousness of gender in sports and physical activities. Journal of Leisure Research, 38, 536-557.

Silva, P., Botelho-Gomes, P., \& Vilodre, S. (2012). Masculinities and sport: the emphasis on hegemonic masculinity in Portuguese physical education classes. International Journal of Qualitative Studies in Education, 25, 269291.

Skelton, C. (2000). 'A passion for football ì: dominant masculinities and primary schooling. Sport, Education and Society, 5, 5-18.

Soler, S. (2009). Los procesos de reproducción, resistencia y cambio de las relaciones tradicionales de género en la Educación Física: el caso del fútbol. Cultura y Educación, 21, 31-42.

Swain, J. (2000). The money ìs good, the fame is good, the girls are good ì: The role of playground football in the construction of young boy ì masculinity in a junior school. British Journal of Sociology of Education, 21, 95- 109.

Swain, J. (2002). The resources and strategies boys use to establish status in a junior school without competitive sport. Discourse: Studies in the Cultural Politics of Education, 23, 91-107.

Swain, J. (2003). Needing to be 'in the know ì: strategies of subordination used by 10- 11 year-old schoolboys. International Journal of Inclusive Education,7, 305-324.

Swain, J. (2006). The role of sport in the construction of masculinities in an English independent junior school. Sport, Education and Society, 11, 317-335.

Velasco, H., \& Díaz De Rada,A. (2009). La lógica de la investigación etnográfica. Un modelo de trabajo para etnógrafos de escuela. Madrid: Trotta.

Vidiella, J., Herráiz, J., Hernández, F., \& Sancho, J. (2010). Deporte, cuerpo y masculinidad(es): constituyendo una masculinidad hegemónica. Movimiento, 16, 93-115.
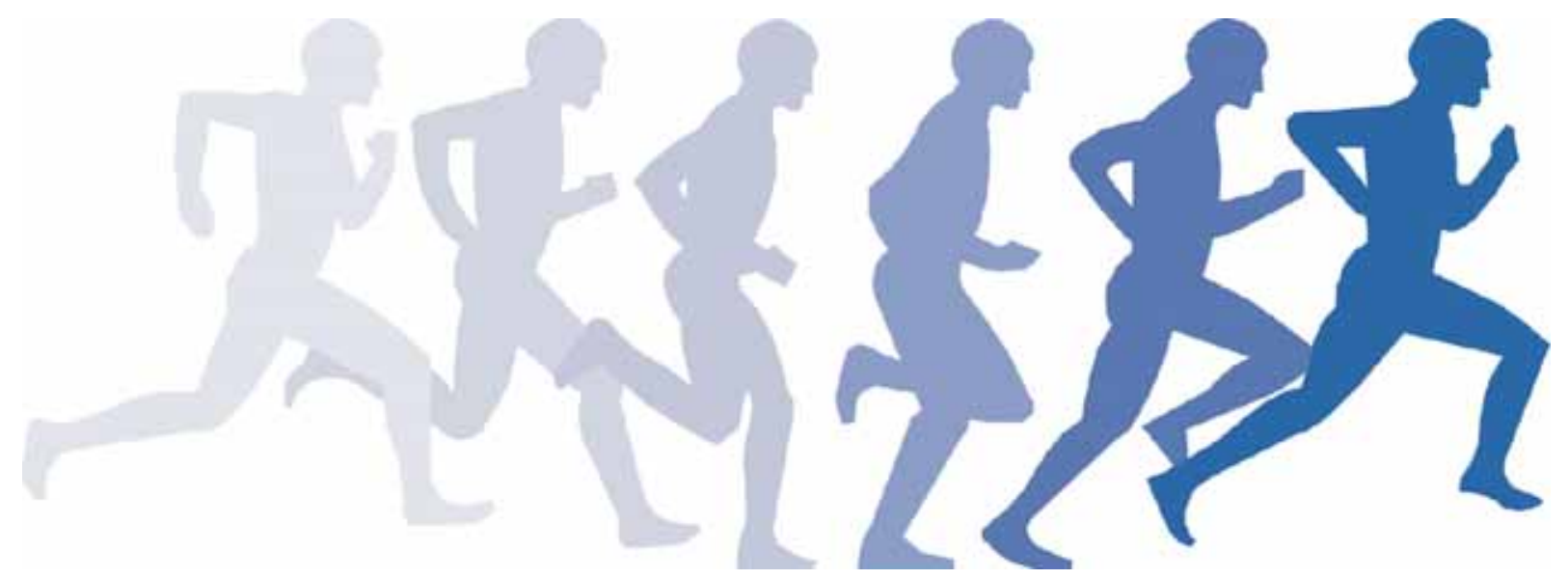